\title{
Estrategia para la gestión de residuos no peligrosos
}

\author{
Strategy for management of no hazardous waste
}

Martha Janneth Saavedra Alemán', Martha Alix Novoa² y Jairo Alberto Ladino ${ }^{3}$

1,2,3 Departamento de Química, Facultad de Ciencia y Tecnología. Universidad Pedagógica Nacional, Bogotá - Colombia.msaavedra@pedagogica.edu.co, marthaalix@gmail.com, dessigna@hotmail.com

\section{Resumen}

En el documento se realiza la descripción de los resultados parciales obtenidos en el proyecto de gestión ambiental: dasificación, cuantificación y disposición de residuos no peligrosos en el Departamento de Química de la Universidad Pedagógica Nacional, cuyo propósito central es diseñar e implementar una estrategia para la gestión integral de los mismos.

Palabras clave

Disposición final de residuos, residuos no peligrosos, recidaje, gestión ambiental, estrategia de acción.

\section{Abstract}

The paper is the description of the partial results obtained in the environmental management project: dassification, quantification and non-hazardous waste disposal at the Chemistry Department at the National Pedagogical University, whose main purpose is to design and implement a strategy for management of the same.

\section{Keywords}

Waste Disposal, non-hazardous waste, recyding, management project, action strategy.

\section{Introducción}

En los últimos años, la preocupación por los problemas ambientales ha sido evidente, debido al impacto negativo que generan tanto en la salud de la población como a nivel de contaminación ambiental. La inadecuada dasificación, manejo, tratamiento y disposición final de los residuos no peligrosos altera el medio ambiente, provocando daños físicos serios a la comunidad en general $\mathbf{y}$, por supuesto al personal involucrado de las instituciones universitarias; todo esto sucede porque no se tiene una cultura y un conocimiento daro hacia el manejo adecuado de los residuos no peligrosos.

Los diferentes proyectos de gestión ambiental analizados, tanto a nivel nacional como internacional, indican que para lograr la ejecución de un programa de control de residuos sólidos se requiere una infraestructura apoyada por un ente gestor que coordine las etapas del proceso en cuanto a recolección, manipulación, almacenamiento, transporte, disposición final, etc. El interés por el tema es evidente pero hay un gran componente legal y normativo que incide en la elaboración del programa de gestión institucional y son muy pocos los que han alcanzado resultados evaluables a partir de la ejecución de las diversas políticas planteadas según las normas, ya que ante todo se requiere de la participación consiente de la comunidad, así como de la orientación hacia nuevas concepciones de residuos, sin dejar de lado que deben ser proyectos sostenibles, controlados y evaluados permanentemente. Por tal razón es de suma importancia que el Departamento de Química de la Universidad Pedagógica Nacional implemente programas para 
concientizar a la comunidad de la adecuada dasificación, manejo, tratamiento y disposición final de los residuos no peligrosos que alteran el medio ambiente, provocando daños físicos serios a la comunidad en general. Los resultados obtenidos con la estrategia propuesta permitirán que la Universidad Pedagógica Nacional analice su participación en este tipo de problemáticas de índole Nacional e Internacional y forme parte en el planteamiento de soluciones.

En el contexto de la investigación se concibe la gestión de residuos como el conjunto de operaciones encaminadas a dar a los residuos sólidos urbanos generados en una determinada zona, el tratamiento global más adecuado, desde los puntos de vista técnico, económico, medioambiental y sanitario, de acuerdo con las características de los mismos y los recursos disponibles. (Colomer, Gallardo, 2007)

Institucionalmente, la Universidad Pedagógica Nacional expidió la resolución 1451 de diciembre de 2008 que establece las condiciones generales para el manejo, recolección, disposición de los residuos sólidos no peligrosos producidos por ésta, según la normatividad establecida por el Ministerio de Salud, el Ministerio de Ambiente, Vivienda y Desarrollo Territorial y la Unidad Administrativa Especial de Servicios Públicos de Bogotá, D.C. El manejo de los residuos comprende las siguientes actividades:

$>$ Almacenamiento o disposición en el punto de generación

$>$ Recolección

$>$ Almacenamiento y disposición temporal

$>$ Transporte

$>$ Tratamiento y desactivación

$>$ Recuperación y recidaje

$>$ Almacenamiento y disposición final

Para el manejo de .los residuos se plantea en la normativa la dasificación según origen en: domésticos, los que por su composición biológica no se pueden combinar con los de tipo recidable y los que por su naturaleza, composición, tamaño y volumen pueden ser incorporados en un proceso espeć́fico de acuerdo con la capacidad de operación. La división de servicios generales es la encargada de la administración, gestión y seguimiento de los procesos y procedimientos relacionados. El programa de manejo de los residuos sólidos no peligrosos induye: Establecimiento de rutas y horarios, los cuales deben ser conocidos por los generadores; mantenimiento de instrumentos, elementos y equipos; capacitación al personal que realiza el manejo y tratamiento de los residuos; actividades a desarrollar en eventos de fallas en el proceso e información a los generadores en cuanto a localización, tamaño, capacidad y calidad de los recipientes.

\section{Desarrollo}

El proyecto de gestión ambiental se encuentra liderado por el grupo de investigación La Didáctica y sus Ciencias dentro de la línea de investigación Incorporación de la Educación Ambiental al Currículo de Ciencias en la Universidad Pedagógica Nacional. En la tabla No.1 se observa la estrategia metodológica propuesta:

\begin{tabular}{|c|l|}
\hline FASES & \multicolumn{1}{c}{ DESCRIPCIÓN } \\
FASE I. & $\begin{array}{l}\text { Realizar un diagnostico (identificación, caracterización y } \\
\text { Cuantificación) de la condición actual de los residuos en el } \\
\text { Departamento de Química de la Universidad Pedagógica Nacional. }\end{array}$ \\
\hline FASEANIZACIÓN & Talleres de inducción y sensibilización. \\
DIVULGACIÓN &
\end{tabular}

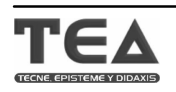


FASE III.

EJECUCIÓN Y SECUIMIENTO

FASE IV.

EVALUACIÓN Y

RETROALIMENTACIÓN
Jomadas de recidaje donde toda la comunidad separará el material reciclable con ayuda de los líderes, donde se realizara un seguimiento y una verificación que permita evaluar la estrategia: monitores

En esta fase se implementará una matriz DOFA que permita analizar los resultados alcanzados hasta el momento y establecerá estrategias de mejoramiento, para ser implementadas a futuro.

Tabla No.1. Estrategia Metodológica.

El proyecto partió del análisis del manejo de los residuos no peligrosos en el Departamento de Química, realizando una caracterización de los mismos, dentro de los resultados obtenidos cabe destacar que el residuo que se genera en mayor proporción es el papel, seguido de envases de plástico, entre otros como: envases de vidrio, vasos de poliestireno, sobrantes de café, guantes de laboratorio y cáscara de frutas. Una vez identificado el residuo que se genera en mayor proporción, se da inicio a las fases II y III siendo los docentes de planta del Departamento de Química la primera población que participó en el programa de manejo de los residuos sólidos no peligrosos. Este programa comprende las siguientes actividades:

- Diseño de colectores para el papel de uso personalizado para el docente

- Establecimiento de rutas de recolección y horarios

- $\quad$ Almacenamiento o disposición en el punto de generación: oficina de cada profesor

- Recolección.

- $\quad$ Almacenamiento y disposición temporal

- Transporte

- Disposición final

- Reciclaje

El programa se ejecutó durante el II semestre del 2008 y a partir de los resultados de la aplicación se construyó una matriz DOFA, la cual se muestra en la Tabla No. 2, con el objeto de determinar si el programa podría ser extensivo a toda la comunidad del Departamento de Química.

\begin{tabular}{|c|c|c|}
\hline & OPORTUNIDADES & AMENAZAS \\
\hline $\begin{array}{l}\text { FACTORES EXTERNOS } \\
\text { FACTORES INTERNOS }\end{array}$ & $\begin{array}{l}\text { 1. Iniciar el proceso de Gestión } \\
\text { Ambiental en el } \\
\text { Departamento de Química } \\
\text { 2. Incorporar al currículo } \\
\text { proceso de educación } \\
\text { ambiental. } \\
\text { 3. Fortalecer la línea de } \\
\text { Investigación en cuanto a } \\
\text { reconocimiento y } \\
\text { experiencia académica. } \\
\text { 4. Plantear una propuesta } \\
\text { innovadora de } \\
\text { concientización en el manejo } \\
\text { de residuos no peligrosos. }\end{array}$ & $\begin{array}{l}\text { 1. Recibir sanciones por } \\
\text { incumplir con la legislación } \\
\text { vigente. } \\
\text { 2. Alteración del medio } \\
\text { ambiente, causando un } \\
\text { impacto negativo a la } \\
\text { comunidad interna y externa } \\
\text { del Departamento. } \\
\text { 3. Resistencia al cambio y } \\
\text { ausencia de una conciencia } \\
\text { colectiva. }\end{array}$ \\
\hline FORTALELAS & ESTRATEGIAS DE FO & ESTRATEGIAS DE FA \\
\hline $\begin{array}{l}\text { 1. El grupo de Investigación } \\
\text { cuenta con Personal } \\
\text { calificado en diferentes } \\
\text { áreas del conocimiento que } \\
\text { consolidan la gestión } \\
\text { ambiental. } \\
\text { 2. El Departamento de Qúmica }\end{array}$ & $\begin{array}{l}\text { 1. Establecer un proceso de } \\
\text { formación ambiental con la } \\
\text { comunidad del } \\
\text { Departamento de Química. } \\
\text { 2. Generar planes de } \\
\text { participación interdisciplinar } \\
\text { en la comunidad. }\end{array}$ & $\begin{array}{l}\text { 1. Realizar actividades de } \\
\text { información y divulgación de } \\
\text { normas y procesos } \\
\text { referentes al proyecto. } \\
\text { 2. Aportar soluciones a } \\
\text { problemáticas en cuanto a la } \\
\text { generación de residuos }\end{array}$ \\
\hline
\end{tabular}


cuenta con Laboratorios

dotados para la

investigación orientada al

tratamiento de residuos.

3. El Departamento de Química está ubicado en una sola planta lo cual facilita la gestión.

DEBILTDADES

1. Interupción de actividades académicas.

2. Recursos económicos deficientes.

3. Ausencia de un proceso continuado en formación ambiental.

4. Inconciencia en la generación de residuos en el individuo.
3. Vincular a la comunidad administrativa y estudiantil al proyecto. sólidos no peligrosos que afecte a la comunidad interna y externa en el Departamento
ESTRATEGIAS DE DO

1. Fomentar jornadas de sensibilización y concientización.

2. Generar estrategias de divulgación y participación que impacten a la comunidad sin uso excesivo de recursos.

3. Desarrollo de trabajos de grado de pregrado y postgrado en Investigación de disposición final de residuos no peligrosos.

Tabla No. 2. Matriz ambiental DOFA Departamento de Química

Con base en la construcción y análisis de la matriz DOFA sugerimos que la sostenibilidad del proyecto no depende de las actividades propuestas a partir de la legislación, sino de la concientización y sensibilización de los residuos producidos por cada individuo y el uso que se le puede asignar a éste. Es importante que el individuo reconozca el impacto y repercusión que producen sus propios residuos sobre sí mismo y su entorno.

La última etapa del proyecto implica hacer extensivo el proyecto a la comunidad del Departamento de Química. La estrategia que se proyecta aplicar está basada en el siguiente supuesto: El individuo de una comunidad asume que existen factores externos que no son importantes y menos aún, si no poseen un beneficio directo, entonces, todo residuo no peligroso que éste genere no será su responsabilidad por lo que no le dará una adecuada disposición final, delegando la responsabilidad a terceros. A partir de lo anterior consideramos que al generar una estrategia de acción, ésta permitirá establecer el grado espećfico de conocimiento y conciencia en los individuos referente al concepto desecho y además permitirá generar una actitud prevaleciente en no ignorar el residuo y su efecto sobre el medio en donde habita o labora (Molano, 2003)

La estrategia de acción propuesta estará constituida por las siguientes etapas: Campaña de expectativa, estimulación y participación; para después de lo cual se esperańa que sean aplicables los lineamientos legales correspondientes a los Planes de Gestión de Residuos.

La etapa de expectativa establecerá una campaña llamada:

\section{Desecha tu inconsciencia \\ DESECHA \\ TU IN CONCIENCIA}

Para esto se generarán carteleras en el Departamento de Química durante 2 semanas cambiando de tema cada 4 días hasta llegar a la última semana, invitando a todos los estudiantes docentes y administrativos del Departamento a conocer sobre el tema de residuos no peligrosos en una campaña de expectativa que permitirá inducir inquietudes respecto al tema propuesto, en este caso conciencia en el uso posterior de los desechos no peligrosos, encaminando a los individuos a 
mantener la inquietud sobre ésta. Esta actividad será complementada por 6 sesiones de cine foro con películas enfocadas al tema de procesos de reciclaje, el uso de residuos no peligrosos y el tema del medio ambiente, publicando previamente las pautas de cada sesión en carteleras y haciendo extensiva la invitación a toda la comunidad del Departamento.

En la etapa de estimulación se diseñará un escenario que se colocará en el techo o en la pared del Departamento de Química, con el fin de que su posición genere una expectativa poco usual y que se recree un espejo de la realidad, con éste se pretende que el individuo vea desde otra perspectiva los elementos dejados en el cotidiano en un espacio de trabajo; el escenario contará con implementos de oficina, desechos de comida y papel dispuestos como si estuviesen en las condiciones que los deja un usuario.

En la etapa de participación se propone lograr junto con los docentes de tiempo completo el uso del salón sin previo mantenimiento por parte del personal de servicios generales para que los estudiantes perciban el impacto que tienen los entes de apoyo.

Es necesario que durante la aplicación de la estrategia se siga manteniendo el sistema de recolección de desechos no peligrosos por la comunidad del Departamento para evaluar la reacción de ellos frente a la estrategia de acción. Y desde allí medir la motivación, el impacto y el uso de los elementos propuestos.

\section{Conclusiones}

Es necesario definir campañas y acciones encaminadas al reconocimiento conciencia e impacto de los desechos frente a una comunidad en sus roles espećficos, estableciendo henramientas de medición del impacto de las mismas sobre el individuo.

Motivar a la comunidad a desarrollar espacios de conocimiento frente a los desechos.

Establecer espacios de disertación frente al tema de los desechos y su impacto en el medio.

Se busca que una vez aplicada la estrategia se defina y se adarare la responsabilidad particular frente a la responsabilidad colectiva al buen uso y manejo de los residuos.

\section{Bibliografía}

Colomer, F.J., Gallardo A. (2007). Tratamiento y gestión de residuos sólidos. Ed. Limusa, Universidad Politécnica de Valencia

Molano C. A. (2003). Desarrollo de la conciencia ambiental por medio de la lúdica. "una propuesta pedagógica desde la educación Ambiental para el desarrollo rural". Lunazul.10-19.

Universidad Pedagógica Nacional (2008). Condiciones generales para el manejo, recolección y disposición de los residuos sólidos no peligrosos. Consejo académico. Resolución 1451, diciembre 10. 